\title{
Estudo comparativo de observação de habilidades sociais de gêmeas com e sem deficiência visual
}

\author{
Carolina Severino Lopes da Costa* \\ Almir Del Prette ${ }^{* *}$
}

\section{Resumo}

O objetivo geral foi descrever e caracterizar o desempenho social de duas crianças, gêmeas idênticas, com dez anos de idade: uma cega e outra vidente. Os dados foram obtidos pela realização de filmagens das crianças em situações livres. As filmagens foram avaliadas por meio de um protocolo de observação das situações livres, de modo que os desempenhos das crianças possibilitaram inferir as classes de habilidades sociais. Os resultados mostraram que a criança vidente apresentou repertório de habilidades sociais mais amplo e refinado do que o de sua irmã. Com base nos dados, pode-se concluir que, neste caso, crianças cegas e videntes possuem repertórios de habilidades sociais distintos, sendo que as diferenças podem estar relacionadas tanto à variável cegueira quanto às diferenças na maneira como o ambiente estimula as crianças.

Palavras-chave: Deficiência Visual. Habilidades Sociais. Irmãos gêmeos.

\section{Comparative study of social skills observation of twin with and without visual impairment}

\begin{abstract}
The general aim was to describe and characterize the social performances of two identical twins aged ten years old: one blind and one sighted. The data were obtained by filming the children during free situations. According to a protocol for observing the free situations, the films were assessed so that the children's performances enabled to infer the categories of social skills. The results showed that the sighted child presented a wider and more refined repertoire of social skills in relation to her sister. From the data, it is possible to conclude, in this case, that blind and sighted children have distinct social skills repertories, and the existing differences can be related to the blind variable as well as to the differences in the way the environment stimulates the children.
\end{abstract}

Keywords: Visual Impairment. Social Skills. Identical Twins.

\footnotetext{
* Professora Doutora da Universidade Federal de São Carlos. Mestre e Doutora em Educação Especial pela Universidade Federal de São Carlos (SP), Professora do Departamento de Psicologia da UNICEP, São Carlos - São Paulo, Brasil.

** Professor Títular da Universidade Federal de Sao Carlos. Com atuação no PPGPSI e PPGEES. São Carlos - São Paulo, Brasil.
} 
A deficiência visual (DV) é um tipo de deficiência sensorial e consiste basicamente em perda total ou parcial do sentido da visão (HALLAHAN; KAUFFMAN, 2009). Com relação ao diagnóstico, a deficiência visual engloba tanto os casos de cegueira como os de baixa visão (visão subnormal/visão parcial) sendo definida sob dois enfoques básicos: clínico e educacional.

Na visão clínica, também denominada legal, a classificação é feita por duas escalas oftalmológicas: de acuidade visual, (o que se vê a determinada distância), e de campo visual (amplitude da área percebida pela visão). É considerado deficiente visual, conforme tabela de Snellen, o indivíduo que tem 20/200 pés de acuidade visual, enquanto usa seus óculos, ou seja, àquele que necessita estar a uma distância de 20 pés ou 6 metros para poder enxergar o que uma pessoa com visão normal enxergaria a 200 pés ou 60 metros (HALLAHAN; KAUFFMAN, 2009; HARDMAN; DREW; EGAN, 2011; HEWARD, 2003).

No enfoque educacional, pode-se dizer que um indivíduo possui deficiência visual quando necessita de instrução em Braille (sistema de escrita por pontos em relevo) ou que lê tipos impressos ampliados, com auxílio de potentes recursos ópticos, como lupa, lunetas, óculos especiais etc. (HALLAHAN; KAUFFMAN, 2009; HARDMAN; DREW; EGAN, 2011). Nesse caso, o impedimento na visão, mesmo com correção, afeta sua desempenho educacional, considerando-se necessária uma reestruturação do ambiente escolar para atender as necessidades educacionais especiais dos deficientes visuais.

Dentro das definições que englobam a perspectiva clínica e educacional da deficiência visual, existem pelo menos duas subdivisões, que caracterizam os casos de cegueira total e de baixa visão. Na cegueira total há completa perda do sentido da visão, embora algumas pessoas consideradas cegas totais possuam percepção de luz (distinção entre claro e escuro) e projeção luminosa, definida pela identificação da direção de onde provém a luz (HARDMAN; DREW; EGAN, 2011).

Nos casos de baixa visão, também chamada visão subnormal /visão parcial, não há completa perda do sentido da visão e o indivíduo utiliza os resíduos visuais na realização de várias atividades. Mas, mesmo assim, o grau da deficiência pode interferir e/ou limitar seu desempenho em vários aspectos como dificuldade ou impedimento no campo visual central, visão embaçada, campo visual restrito, visão em tubo etc (BATISTA, 1998).

As diferenças entre a percepção de crianças cegas e videntes implicam em desenvolvimento e aprendizagens distintos (OCAHITA; ROSA, 1995) e, embora a literatura aponte diferenças em várias áreas do desenvolvimento infantil (KEKELIS, 1997; OCHAITA; ROSA, 1995), este estudo tem como enfoque o desenvolvimento social, particularmente o desempenho social.

De acordo com Del Prette e Del Prette (2001), o desenvolvimento social se dá principalmente na interação recíproca entre o indivíduo e seu ambiente sociocultural. Este "pode ser entendido como o conjunto de modificações 
que ocorrem na complexidade e natureza das relações e interações com as outras pessoas e, concomitantemente, nos processos cognitivos, afetivos e conativos a elas associados" (DEL PRETTE; DEL PRETTE, 2001, p. 38).

Diferentes teorias da aprendizagem explicam as aquisições efetuadas pelo organismo humano. Além da aprendizagem pela conseqüência e instrução, as aquisições novas ocorrem via imitação. O modelo teórico da aprendizagem social entende que o desenvolvimento social ocorre por meio da observação do comportamento de outras pessoas, processo este denominado vicário (BANDURA, 1979). Del Prette e Del Prette (1999) acrescentam que para ocorrer o processo de modelação, o indivíduo deve ser exposto ao modelo para apreender o desempenho observado, o que consequentemente, exige a utilização dos sentidos visão e audição. A descrição da situação estímulo feita por meio de mediação verbal e auditiva é outro recurso utilizado no processo de aprendizagem (DEL PRETTE; DEL PRETTE, 2002) e deve ser empregado, de modo especial, com indivíduos que possuem deficiência visual.

Com relação ao desenvolvimento social de crianças com deficiência visual Hardman, Drew e Egan (2011) afirmam que a capacidade de crianças com perda de visão para se adaptar ao ambiente social depende de uma série de fatores, tanto hereditário como experiencial. A ausência de visão altera a percepção que o indivíduo tem de si mesmo e dos demais, podendo ser drasticamente distorcida. Crianças com perda de visão têm menor probabilidade de iniciar uma interação social e menos oportunidade de socializar-se com outras crianças. Ainda, de acordo com os autores, elas são muitas vezes incapazes de imitar os maneirismos físicos dos outros e, portanto, não desenvolvem um componente muito importante de comunicação social: a linguagem do corpo.

As sutilezas da comunicação não verbal podem alterar significativamente o significado pretendido das palavras faladas e a incapacidade de uma pessoa de aprender e usar pistas visuais (tais como expressões faciais e gestos com as mãos) tem profundas conseqüências para as interações interpessoais (HARDMAN; DREW; EGAN, 2011). A pessoa com perda de visão nem pode ver, os elementos visuais que acompanham as mensagens recebidas de outros, nem tampouco consegue captar o sentido das mensagens a ser transmitido pela linguagem corporal. Por outro lado, Hardman, Drew e Egan (2011) acrescentam que a ausência do uso da visão leva a exclusão de algumas atividades como a dos esportes e também a restrição de experiências com imagens (cinema, televisão, museu), dificultando as interações sociais com os videntes e reforçando a noção equivocada de falta de interesse por essas atividades.

Ainda que haja diferenças no curso do desenvolvimento social entre videntes e não videntes, não se pode afirmar que o aspecto orgânico da deficiência seja impeditivo de um desenvolvimento social adequado, mas que diferentes processos de aprendizagem (fatores ambientais) podem favorecer o desenvolvimento de pessoas com deficiência (CUNHA; ENUMO, 2003; VIGOTSKY, 1997). O desenvolvimento de habilidades sociais em pessoas cegas deve, por 
um lado, se ajustar às possibilidades de representação e ação que os canais sensoriais Ihes permitem e, por outro de estimulação diferencial que recebem de seu entorno. Além disso, é possível explorar de várias maneiras recursos tecnológicos disponíveis, por exemplo o computador, permitindo e ampliando o acesso de deficientes visuais a outras experiências.

Quando se avalia um repertório de habilidades sociais, é importante avaliar déficits e excessos comportamentais (DEL PRETTE; DEL PRETTE, 2005). Os déficits em habilidades sociais, conforme Del Prette e Del Prette (2005) podem ser agrupados em três tipos: a) Déficit de aquisição, que se refere a uma ou mais habilidades não aprendidas e que, por isso, encontram-se ausentes no repertório da pessoa; b) Déficit de fluência, que se caracteriza pelo desempenho que não atinge os níveis de proficiência esperados, seja por falhas no timing, nos componentes não verbais ou nos conteúdos verbais que definem essa proficiência; e c) Déficit de desempenho, consiste da emissão de desempenhos socialmente competentes em freqüência inferior à esperada pelo ambiente.

Sabe-se que os déficits em habilidades sociais estão associados à pior qualidade de vida, dificuldades interpessoais e diversos transtornos psicológicos como depressão, isolamento social, timidez, abuso de drogas, entre outros (DEL PRETTE; DEL PRETTE, 1999; 2001).

Outro ponto a ser destacado remete ao fato de que a maioria dos estudos realizados com crianças cegas não permite a possibilidade de controlar a variabilidade genética, que pode influenciar as diferenças encontradas no repertório de habilidades sociais, além dos fatores ambientais associados. Estudos com gêmeos idênticos, que compartilham o mesmo ambiente familiar, têm sido considerados um método privilegiado para examinar influências genéticas e ambientais nas diferenças individuais de comportamento (OLIVER; PLOMIN, 2007). Com base nessas considerações, o presente estudo investiga o repertório de habilidades sociais de duas crianças gêmeas idênticas, uma com deficiência visual e outra sem esse comprometimento. A pesquisa obteve aprovação do Comitê de Ética, pelo número do processo 125/3.

\section{Objetivos}

- Descrever e caracterizar o desempenho social de uma criança cega e uma vidente, gêmeas idênticas.

-Caracterizar semelhanças e diferenças entre classes de habilidades sociais inferidas a partir do repertório de desempenho social das duas irmãs.

\section{Método}

\section{Participantes}

Participaram da pesquisa duas crianças do sexo feminino, gêmeas idênticas, com idade de 10 anos, uma cega, Célia, e outra vidente, Virna (nomes 
fictícios). As duas crianças, na ocasião da coleta de dados, cursavam a $3^{\mathrm{a}}$ série do Ensino Fundamental em escolas públicas do município. Célia participava, ainda, da sala de recursos para deficientes visuais de uma escola municipal. As duas crianças nasceram prematuras e permaneceram na incubadora por dois meses. Célia teve retinopatia da prematuridade, apresentando o diagnóstico de cegueira congênita. As duas crianças apresentavam características fenotípicas semelhantes (altura, peso, cor do cabelo e pele), exceto que Célia havia quebrado um dos dentes devido a uma queda acidental.

\section{Local}

A coleta de dados foi realizada na casa dos participantes, em um bairro de nível sócio-econômico baixo de uma cidade, do interior do estado de São Paulo, com aproximadamente 250 mil habitantes.

\section{Materiais e Instrumentos}

Foram utilizados: a) Câmera VHS gradiente GCP-195; b) Protocolo de observação das filmagens.

\section{Procedimento de Coleta de Dados}

Foram realizadas quatro visitas para que as crianças se familiarizassem com a pesquisadora (raport). Após esse período agendou-se com a família a realização de filmagens das crianças. Foram feitas gravações de várias situações nos períodos da manhã e tarde, de um dia de férias das crianças. Essas situações eram: 1) alimentação (1.a. café da manhã/ 1.b. almoço); 2) higiene (2.a. escovar dentes); 3) brincadeiras (3.a. andar de bicicleta/ 3.b. correr/ 3.c. cantar e dançar) 4) solicitação da mãe (4.a.fazer compras). A duração total da filmagem foi de 60 minutos.

\section{Procedimento de análise dos dados}

As situações filmadas foram transcritas e, posteriormente, registradas no protocolo de observação da filmagem, contendo a identificação da situação (alimentação, higiene, brincadeiras ou solicitação da mãe), uma breve descrição do contexto, um quadro com a especificação de cada criança por meio do nome fictício, espaço para registrar os desempenhos de cada criança os antecedentes e, ainda, uma seção para registrar as classes de habilidades sociais inferidas.

A inferência das classes de habilidades sociais de cada criança foi feita mediante análise de seus desempenhos e antecedentes, baseada em definições existentes de diferentes classes (componentes) de habilidades sociais (AGUIAR, 2003; DEL PRETTE; DEL PRETTE, 1999, 2001; HILDEBRAND, 2000). Ao todo, inferiu-se 25 classes de habilidades sociais dos desempenhos de Célia e Virna. As classes de habilidades sociais foram submetidas à avaliação de dois pesquisadores que avaliaram a adequação entre o desempenho e a nomeação dada (inferência das habilidades sociais). 


\section{Resultados e discussão}

Apresenta-se Inicialmente uma tabela contendo as classes de habilidades inferidas dos desempenhos de Célia e Virna.

Tabela 1 - classes de habilidades sociais inferidas dos desempenhos de Célia e Virna

\begin{tabular}{|c|c|c|}
\hline \multirow[t]{2}{*}{ CLASSES DE HS } & \multicolumn{2}{|c|}{ CRIANCAS } \\
\hline & Célia & Virna \\
\hline Advertir & $\bullet$ & $\bullet$ \\
\hline Dar ordens & • & • \\
\hline Atender ordens & • & • \\
\hline Fazer perguntas & $\bullet$ & $\bullet$ \\
\hline Responder perguntas & $\bullet$ & $\bullet$ \\
\hline Fazer pedidos & - & - \\
\hline Recusar pedidos & $\bullet$ & $\bullet$ \\
\hline Expressar desagrado & $\bullet$ & - \\
\hline Expressar desconhecimento & $\bullet$ & - \\
\hline Defender-se & $\bullet$ & $\bullet$ \\
\hline Defender direitos (dos outros) & - & $\bullet$ \\
\hline Explicitar conseqüência & $\bullet$ & - \\
\hline Fornecer instruções & - & $\bullet$ \\
\hline Demonstrar (fornecer prompt) & - & • \\
\hline Seguir demonstração & $\bullet$ & - \\
\hline Fazer comentário & - & - \\
\hline Fazer referência a si mesmo & - & • \\
\hline Ceder & - & $\bullet$ \\
\hline Cooperar & - & $\bullet$ \\
\hline Consolar & - & $\bullet$ \\
\hline Estabelecer contato visual & - & $\bullet$ \\
\hline Convidar & $\bullet$ & $\bullet$ \\
\hline Aceitar convite & $\bullet$ & $\bullet$ \\
\hline Estipular regra & - & $\bullet$ \\
\hline Negociar & - & - \\
\hline
\end{tabular}

Onde: ( ) Habilidade social observada/ (-) Habilidade social não observada 
Considerando o contexto avaliado (ambiente familiar) e a forma como os dados foram obtidos (filmagens), pode-se afirmar que a maioria das classes de habilidades sociais relacionadas é comum a ambas as crianças, porém não obstante as crianças compartilharem de estimulação ambiental muito semeIhante, algumas classes de habilidades sociais registradas diferem entre as duas.

Célia apresentou um número considerável de classes de habilidades sociais, porém apenas quatro específicas que foram as de expressar desagrado, explicitar conseqüência, expressar desconhecimento e seguir demonstração. Considera-se de grande importância que a classe denominada seguir demonstração esteja presente no repertório de Célia, o que pode favorecer o aprendizado de novos comportamentos, se exposta a situações que favoreçam novas aquisições e/ou possam refinar o repertório existente.

Observa-se, ainda, a presença das classes de habilidades sociais de recusar pedidos, expressar desagrado e defender-se, consideradas habilidades sociais assertivas (DEL PRETTE; DEL PRETTE, 2001) relevantes para Célia, que se encontra em idade escolar, frequentando, por sua vez, ambientes fora do contexto familiar (escola regular, sala de recursos) que demandam desempenhos de autoproteção. Entretanto, não basta a presença de tais habilidades no repertório do indivíduo, mas a competência para usá-las de forma apropriada e no contexto adequado, discriminando os mais variados tipos de interlocutores e, ainda, as formas apropriadas das mensagens não verbais (DEL PRETTE; DEL PRETTE, 1999; 2001; HARDMAN: DREW; EGAN, 2011).

A utilização das filmagens, por exemplo, é um recurso metodológico privilegiado por possibilitar o acesso aos componentes não verbais $\mathrm{e}$ paralinguísticos das interações sociais, todavia tal recurso ainda não é utilizado em larga escala com deficientes visuais. Conforme sinaliza Del Prette e Del Prette (2011), reconhecer e regular tais componentes "associados a determinadas classes de habilidades sociais pode ampliar a competência social do indivíduo para lidar com as demandas de uma dada situação" (p. 173).

Algo que chama atenção nas falas de Célia é a forma como apresenta suas frases, por exemplo: Não vai aí, vai para lá!!; Você não vai!!!. Tais aspectos paralingüísticos presentes na comunicação de Célia (tom de voz elevado associado ao uso de frases imperativas) são indicadores do que se poderia considerar uma certa rudeza na classe intitulada dar ordens. Contudo, é provável que, por não dispor de feedback visual do comportamento dos interlocutores, Célia desenvolveu um estilo confrontativo e autoritário que, certamente, tem sido funcional.

Com relação às classes de habilidades sociais presentes no repertório específico de Virna, merecem destaque as de negociar, ceder e consolar (essas duas últimas podem ainda ser inseridas em uma classe mais ampla denominada de habilidades socais empáticas), muito valorizadas nos relacio- 
namentos com irmãos, pais, amigos e podem se constituir em facilitadores para obtenção de maior aceitação pelos pares, indicação de preferência por amigos, elogios pelos pais (DEL PRETTE; DEL PRETTE, 2005; FERREIRA; DEL PRETTE; LOPES, 2009; FALCONE, 2004; GODOY, 2007). Sabe-se que determinadas classes de habilidades sociais podem gerar maior número de consequências positivas para o indivíduo que os emite com eficácia (JUNQUEIRA, 2011) e, portanto, aumentar as oportunidades para novas aprendizagens.

Somando-se a isso, considera-se importante ressaltar a presença das classes de habilidades sociais de fornecer instruções, demonstrar e cooperar presentes no repertório de Virna. Esse aprendizado está, provavelmente, associado às consequências positivas liberadas pelo ambiente, principalmente a mãe, quando esta lidava com a irmã. Essas habilidades sociais foram importantes tanto para Virna como para Célia, pois Virna pode se constituir em importante agente socializador da irmã, conforme destaca a literatura corrente (KEKELIS, 1997) em que os irmãos podem ser significativos agentes de socialização uns dos outros.

É interessante acrescentar que os programas de Treinamento de Habilidades Sociais dão ênfase nas aprendizagens de comportamentos funcionais (ANZANO; RUBIO, 1995; CABALLO, VERDUGO; DELGADO, 1997; DEL PRETTE; DEL PRETTE, 2003; FREITAS ET AL., 1999; GRESHAM, 2011), considerando que as habilidades não valorizadas não se mantêm no ambiente.

Pode-se dizer, de modo geral, que Virna apresentou repertório de habilidades sociais mais amplo e diversificado que Célia, o que seria esperado. Somando-se a isso, dentro das classes de habilidades sociais consideradas comuns às duas crianças, foi possível identificar diferenças quanto à qualidade dos desempenhos emitidos por elas, podendo-se dizer que os desempenhos de Virna atingiram maior nível de proficiência.

Em seguida será apresentada uma síntese de possíveis déficits em habilidades sociais apresentados por Célia e Virna com base na avaliação dos desempenhos de ambas nas sete diferentes situações descritas anteriormente e na literatura da área (DEL PRETTE; DEL PRETTE, 2005), conforme a Tabela 2. 
Tabela 2 - Déficits em habilidades sociais de Célia e Virna nas quatro diferentes situações

\begin{tabular}{|c|c|c|c|c|c|c|}
\hline Criança & Situação & Desempenho contextualizado & $\begin{array}{l}\text { Classe } \\
\text { de HS }\end{array}$ & \multicolumn{3}{|c|}{ Déficits } \\
\hline & & & & $A$ & $\mathrm{~F}$ & D \\
\hline Célia & 1 & $\begin{array}{c}\text { Célia percebe a presença de } \\
\text { cebola em seu prato e diz: Mãe } \\
\text { eu não quero cebola }\end{array}$ & $\begin{array}{l}\text { Express- } \\
\text { ar } \\
\text { desagra- } \\
\text { do }\end{array}$ & & $x$ & \\
\hline Célia & 2 & $\begin{array}{l}\text { Virna solicita a vez para brincar. } \\
\text { Célia continua pedalando a } \\
\text { bicicleta e nada responde à irmã }\end{array}$ & $\begin{array}{l}\text { Recusar } \\
\text { pedidos }\end{array}$ & & $x$ & $x$ \\
\hline Virna & 3 & $\begin{array}{l}\text { Virna tenta mostrar à Célia como } \\
\text { se liga o aparelho de som. Tem } \\
\text { que apertar uma vez só o botão, } \\
\text { assim oh: aperta com o dedo } \\
\text { indicador o botão play uma vez }\end{array}$ & & & $x$ & \\
\hline Célia & 4 & $\begin{array}{l}\text { Célia solicita acompanhar Virna } \\
\text { nas compras e diz à mãe: Eu } \\
\text { quero ir mãe! Deeixa!! Eu quero } \\
\text { ir mainhê!!! }\end{array}$ & & & $x$ & \\
\hline
\end{tabular}

$A$ : Aquisição; F: Fluência e $D$ : Desempenho

1: Almoço; 2: Andar de bicicleta; 3: Cantar e dançar; 4: Fazer compras

Nesse quadro é possível inferir que Célia e Virna não apresentaram déficit de aquisição e possivelmente apenas uma ocorrência de déficit de desempenho apresentada por Célia na classe recusar pedidos. Virna e Célia apresentaram em comum apenas déficit de fluência, porém em classes de habilidades sociais diferentes. sociais:

Pode-se dizer que Célia apresentou déficit de fluência nas habilidades

a) recusar pedidos, pois não emitiu nenhum desempenho verbal associado ao comportamento de permanecer pedalando a bicicleta, o que era esperado de ocorrer. Nessa situação ela, portanto, não explicitou a negativa (Não), nem mesmo as razões pelas quais recusou o pedido de Virna. Ela sequer propôs uma negociação como, por exemplo: "vou dar mais cinco voltas e depois cedo a bicicleta a você". 
b) fazer pedidos, os aspectos paralingüísticos das verbalizações de Célia (Eu quero ir mãe. Mãe eu quero ir. Deeixa!!!! Eu quero ir mainhể”) permitem classificá-los como comportamentos de birra, resultando em negativa por parte da mãe e, portanto, não alcançando o objetivo na situação de Célia ir com a irmã às compras, mas que eventualmente obtinham resultados positivos. Ainda que a consecução de objetivos em uma situação de interação social seja um indicador de competência social (DEL PRETTE; DEL PRETTE, 2001), outros critérios são, igualmente, importantes e devem ser vistos em seu conjunto.

c) expressar desagrado, ao perceber a presença de cebola em seu prato, seria esperado que ela solicitasse à mãe ou à irmã que uma delas a retirasse de seu prato. Nesse caso ela apenas afirmou que não queria ingerir as cebolas.

Virna, por sua vez, apresentou déficit de fluência na classe de habilidades sociais denominada demonstrar. Porém, se considerássemos a comunidade visual como interlocutor de Virna na situação da brincadeira cantar e dançar, o desempenho de Virna, em que se infere a classe de habilidades sociais demonstrar, não pode se enquadrar nesta lista de déficits.

No entanto, considerando que na situação em questão, o interlocutor de Virna era sua irmã, que apresenta a característica peculiar de não enxergar, a complementaridade não verbal fornecida por Virna não foi acessível à Célia, não atingindo o possível objetivo de ensinar Célia a manipular o botão playcorretamente.

Essa dificuldade pode estar ligada ao fato de os dados sugerirem ainda que a comunidade visual (pais, cuidadores, irmãos, colegas), via de regra, organiza seus procedimentos educativos e interações com a criança cega de modo semelhante que atua em relação à criança vidente. Os feedbacks não verbais (balançar a cabeça, o sorriso, os gestos que complementam informações) quase sempre estão presentes nas interações dos cuidadores com o deficiente visual, mas na maioria dos casos não são acessados por ele.

\section{Conclusão}

Os resultados obtidos com este trabalho permitem algumas conclusões importantes sobre o conhecimento da criança deficiente visual e da vidente. Pode-se dizer, de forma geral, considerando o contexto avaliado, que foram obtidas informações relevantes sobre diferenças e semelhanças de classes de habilidades sociais presentes no repertório de Célia e Virna, o que permite uma orientação mais focal com o cuidador e/ou treino específicos para o deficiente visual.

A despeito dos dados se restringirem apenas ao contexto doméstico há uma forte evidência de desequilíbrio de repertório de habilidades sociais entre as duas crianças gêmeas idênticas, considerando que ambas compartilham o 
mesmo ambiente e experiências de aprendizagem muito semelhantes nesse contexto. Os dados indicam que a criança vidente, Virna, possui repertório de habilidades sociais mais amplo e elaborado que sua irmã deficiente visual. Esses dados estão de acordo com a literatura corrente (FARRENKOPF, 1995; OCHAITA; ROSA, 1995) que afirma haver diferenças no desenvolvimento social de crianças deficientes visuais e videntes. É importante destacar que tais diferenças podem revelar a necessidade de uma estimulação diferenciada por parte da família por exemplo, para a criança deficiente visual.

Provavelmente Célia e Virna apresentem outras classes de habilidades sociais não observadas no contexto familiar. Além disso, os dados do presente estudo são provenientes de apenas um dia de observação direta via filmagem, tal ressalva deve ser considerada quanto às afirmações acerca do repertório de habilidades sociais das duas crianças em função do curto período de observação.

Adicionalmente deve-se considerar o fator limitante de o presente trabalho ter priorizado o método de observação direta de situações livres com as crianças, portanto as classes de habilidades sociais obtidas foram apenas aquelas que puderam ser inferidas dos desempenhos que as crianças emitiram livremente. Com relação a isso, poder-se-ia utilizar procedimentos diferentes como por exemplo, com a utilização de situações estruturadas denominadas vivências (DEL PRETTE; DEL PRETTE, 2001; 2005) que criassem demandas específicas para a emissão de diferentes classes de habilidades sociais pelas meninas. Outra alternativa seria a de observações em situações estruturadas (DEL PRETTE; PEREIRA, 2008; PEREIRA, 2010).

Constatando-se o baixo número de pesquisas sobre habilidades sociais com indivíduos cegos no Brasil, pode-se considerar que este campo de estudo precisa receber maior atenção, e que muitas questões estão, ainda, requerendo investigação. Dentre elas pode-se destacar, por exemplo, o estudo longitudinal poderia trazer informações importantes sobre a aprendizagem de habilidades sociais das irmãs gêmeas. De maneira similar ao que já foi feito com outra população (Pereira, 2010), programas de treinamento de habilidades sociais para crianças com deficiência visual seriam também altamente indicados.

\section{Referências}

AGUIAR, A. A. R. Análise das habilidades comunicativas de adultos portadores de retardo mental. 2003. 92f. Dissertação. (Mestrado em Educação Especial) - Programa de Pós-Graduação em Educação Especial. Universidade Federal de São Carlos, São Carlos.

ANZANO, S. M.; RUBIO, J. M. L. Entrenamiento en habilidades sociales a niños con ceguera congénita. In: RODRÍGUES, F. G; RUBIO, J.M.L; EXPÓSITO, L. J. (Orgs.). Habilidades Sociales y Salud. Madrid: Ediciones Pirámides, p. 147-183, 1995. 
ARÁNDIGA, A. V.; TORTOSA, C. V. Las habilidades sociales en la escuela: una propuesta curricular. Madrid: EOS, 1996.

BANDURA, A. Modificação do comportamento. Rio De Janeiro: Interamericana, 1979.

BATISTA, C. G. Crianças com deficiência Visual - como favorecer sua escolarização? Temas em Psicologia, Campinas, v. 26, n. 2, p.217-229, 1998.

CABALLO, C.; VERDUGO, M. A. Habilidades sociales en personas con deficiencia visual. In:I JORNADAS CIENTÍFICAS DE INVESTIGACIÓN SOBRE PERSONAS CON DISCAPACIDAD. Anais da I jornadas científicas de investigación sobre personas con discapacidad. Madrid, EOS, v. 19, p. 43$69,1995$.

CABALLO, C., VERDUGO, M.A.; DELGADO, J. Un programa de entrenamiento en habilidades sociales para alumnos con deficiencia visual. In:II JORNADAS CIENTÍFICAS DE INVESTIGACIÓN SOBRE PERSONAS CON DISCAPACIDAD. Anais da II jornadas científicas de investigación sobre personas con discapacidad. Madrid, EOS, v. 16, p. 67-81,1997.

CUNHA, A. C. B; ENUMO, S. R. F. Desenvolvimento da criança com deficiência visual (DV) e interação mãe-criança: algumas considerações. Psicologia, Saúde \& Doenças, v. 4, n. 1, p. 33-46, 2003.

DEL PRETTE, A; DEL PRETTE, Z. A. P. Psicologia das relações interpessoais: vivências para o trabalho em grupo. Petrópolis: Vozes, 2001.

DEL PRETTE, A; PEREIRA, C. S. Procedimento de observação em situações estruturadas para avaliação de habilidades sociais de adolescentes. Psicolog, v. 1 , n. 1 , p. $55-67,2008$

DEL PRETTE, Z. A. P; DEL PRETTE, A. Psicologia das habilidades sociais na infância: Teoria e prática. Petrópolis: Vozes, 2005.

. Habilidades sociais e dificuldades de aprendizagem: Teoria e pesquisa sob um enfoque multimodal. In: DEL PRETTE, A; DEL PRETTE, Z.A.P. (Orgs.). Habilidades sociais, desenvolvimento e aprendizagem: questões conceituais, avaliação e intervenção. Campinas: Alínea, p. 167-200, 2003.

Psicologia das habilidades sociais: terapia e educação. Petrópolis: Editora Vozes, 1999.

FALCONE, E. A evolução das habilidades sociais e o comportamento empático. In: SILVARES, E. F. M. (Org.). Estudos de caso em psicologia clínica comportamental infantil. Campinas: Papirus, p. 49-77, 2004.

FALCONE, E. O. Contribuições para o treinamento de habilidades de interação. In: GUILHARDI, H. J; MADI, M. B. B. P; QUEIROZ, P. P; SCOZ, M.C. (Orgs.).Sobre comportamento e cognição: contribuições para a construção da teoria do comportamento. Santo André, SP: ESETec, p. 91-104, 2002. 
FARRENKOPF, C. Development for preschool children with visual impairments. Annual International Convention of the Council for Excepcional Children. Indianápolis: Texas, p. 11-17, 1995.

FERREIRA, B. C; DEL PRETTE, Z. A. P; LOPES, D.C. Habilidades empáticas de crianças videntes e cegas e a possível influência de variáveis sociodemográficas. Interação em Psicologia, v. 13, n. 1, p. 49-58, 2009.

GODOY, A. M. A. Habilidades sociais empáticas em crianças com deficiência visual. 126f. 2007. Dissertação de Mestrado apresentada ao Programa de Pós-Graduação em Educação Especial - UFSCar, São Carlos/SP.

GRESHAM, F. M. Análise do comportamento aplicada às habilidades sociais. In: DEL PRETTE, A; DEL PRETTE, Z. A. P. (Orgs.). Psicologia das habilidades sociais: diversidade teórica e suas implicações. 2.ed. Petrópolis: Vozes, p.17-66, 2011.

HALLAHAN, D. P; KAUFFMAN, J. M. Exceptional learners: an introduction to special education. Eleven Edition. Needham Heights, MA: Allyn \& Bacon, 2009.

HARDMAN, M. L.; DREW, C. J.; EGAN, M. W. Human exceptionality: school, community, and Family. Tenth Edition. Belmont, CA: Wadsworth, 2011.

HEWARD, W. L. Exceptional Children: an introduction to special education. 7. ed. United States, 2003.

HILDEBRAND, F.C. Dificuldades de aprendizagem: habilidades sociais presentes nas interações. 2000. 92f. Dissertação. (Mestrado em Educação Especial) - Programa de Pós-Graduação em Educação Especial. Universidade Federal de São Carlos, São Carlos/SP.

KEKELIS, L. S. Peer interaction in childhood: the impact of visual impairment. In: SACKS, S.Z; KEKELIS, L.S; GAILORD-ROSS, R.J. (Orgs.).The development of social skills by blind and visually impaired students. 2 . ed. New York: American Fundation for the Blind, p. 13-35, 1997.

NEWCOMBE, N. Desenvolvimentoinfantil: abordagem de Mussen. (Trad. C. Buchweitz), 8. ed, Porto Alegre: Artes Médicas Sul, 1999.

OCHAITA, E; ROSA, A. Percepção, ação e conhecimento em crianças cegas. In: COLL, C; PALACIOS, J; MARCHESI, S.M. (Orgs). Desenvolvimento psicológico e educação: necessidades educativas especiais. (Trad. M. A. G. Domingues). Porto Alegre: Artes Médicas, vol. 3, 1995. p. 318-345, 1995.

OLIVER, B. R; PLOMIN, R. Twins' Early Development Study (TEDS): Multivariate, Longitudinal Genetic Investigation of Language, Cognition and Behavior Problems from Childhood Through Adolescence. Twin Research and Human Genetics, v. 10, n. 1, p. 96-105, 2007. 
PEREIRA, C. S. Programa de habilidades sociais profissionais para pessoa com deficiência física desempregadas: necessidades, processo e efeitos, 2010, 239 f. Dissertação (Doutorado em Educação Especial). Programa de Pós Graduação Em Educação Especial. Universidade Federal de São Carlos. São Carlos/São Paulo.

VYGOTSKY, L. S. Obras escogidas, V- fundamentos de defectología. Trad. J. G. Blank. Madri: Ed. Visor, 1997.

\section{Correspondência}

Carolina Severino Lopes da Costa - Rua Manoel Wenzel, 105 - Vila Madre Cabrini, CEP: 13572-510 - São Carlos, São Paulo, Brasil.

E-mail: caroline_costa@yahoo.com.br - adprette@ufscar.br

Recebido em 09 de agosto de 2011

Aprovado em 16 de novembro de 2011 